\title{
STRUCTURAL TESTS OF FREIGHT WAGONS ON THE BASIS OF SIGNALS GENERATED BY PIEZOELECTRIC MACRO FIBER COMPOSITES
}

\author{
Marek PŁACZEK*, Andrzej WRÓBEL*, Andrzej BUCHACZ* \\ ${ }^{*}$ Institute of Engineering Processes Automation and Integrated Manufacturing Systems, Faculty of Mechanical Engineering, \\ Silesian University of Technology, ul. Konarskiego 18A, 44-100 Gliwice, Poland \\ marek.placzek@polsl.pl, andrzej.wrobel@polsl.pl,andrzej.buchacz@polsl.pl
}

received 10 October 2016, revised 16 September 2017, accepted 19 September 2017

\begin{abstract}
Paper presents a report of a research work that concerns possibilities of freight wagons modernization using new composite materials. The main aim of presented work was to verify the possibility of inference from the dynamic response of the wagon about the changes in its technical condition. During the presented works tests on real objects were carried out using Macro Fiber Composite (MFC) piezoelectric transducers glued to the freight wagon's frame. The dynamical response of the wagon was measured while the object was driving. On the next stage the measured signal was generated on a laboratory stand using electrodynamic modal shaker and vibrations of the laboratory model were measured. Measured signals were juxtaposed on charts and analysed. The aim of this work was to verify if it is possible to detect the change in the system using measurements of vibrations that are being generated during exploitation of the freight wagon.
\end{abstract}

Keywords: Dynamic Response, Freight Wagon, Piezoelectric Transducers, Smart Materials, Analysis of Vibration

\section{INTRODUCTION}

The aim of presented research work was to modernize the analysed wagon during its renovation using new materials. This work is a continuation of the previous research works concerned with analysis of new, composite materials application in freight wagons (Wrobel et al., 2015; Baier and Zolkiewski, 2013; Placzek et al., $2015 \mathrm{a}, \mathrm{b})$. Effects that are to be achieved by the modernization are better corrosion protection of the wagon elements, easier unloading of the wagon in winter conditions (no freezing of the cargo to the sides and floor of the wagon), reduction of the weight of the wagon while its load increases and easier management of freight wagons during exploitation. The 4-axle open wagon series EAOS type 1415 A3 produced by BREC Belgium is being taken into consideration because it is one of the most popular type of wagons designed to unload with the use of tipplers.

An opportunity to apply new materials and new methodology allows designing devices and systems that, for example are more effective, have better properties and lower costs of production. This is why a lot of effort is spent on development of any types of devices that are used (Jamroziak, 2013; Rusinski et al., 2012; Tuma, 2009; Jamroziak et al., 2013). Smart materials, so the materials that can change one or more of their properties during operations and this change can be controlled bring a lot of new possibilities as well. Piezoelectric transducers which are widely used in many kinds of technical devices and number of their applications are still increasing are also smart materials. They properties caused that they can be used in large variety of applications, for example in vibration damping or excitation, energy harvesting systems, as sensors or in order to obtain precise elements positioning (Tuma et al., 2011; Placzek, 2015; Buchacz et al., 2014, 2015).

In 1996 Macro Fiber Composites (MFC) was invented by NASA. MFC transducer is consists of rectangular piezo ceramic rods sandwiched between layers of adhesive, electrodes and polyimide film. Main benefits of the MFC are: increased strain actuator efficiency, damage tolerance, environmentally sealed packages, available as elongators and contractors (Placzek et al., 2015b; Buchacz et al., 2015). The development of transducers allows new applications of piezoelectric materials and improves operation of existing devices.

In this work some benefits obtained by using new piezoelectric transducers application is presented. In previous works for example efficiency of both PZT and MFC transducers used as actuators of the mechanical system was presented. An analysis of the considered mechatronic system was done using a discretecontinuous mathematical model and an approximate method presented in previous publications (Placzek, 2015; Buchacz et al., 2014, 2015). On the other hand, computer aided methods of designing, manufacturing and product life cycle management are also powerful tools that helps to design and produce modern technical devices (Paprocka et al., 2014; Banas et al., 2015; Monica, 2015; Klarecki et al., 2015). Modern systems include elements from different science areas, such as mechanics, electronics and informatics. Such connection brings new possibilities and new effects, so those systems can be called mechatronic systems (Buchacz and Galeziowski, 2012; Gwiazda et al., 2015).

Rail transport is a very important part of the modern economy, one of the components determining its dynamic development. It is therefore important to conduct research and taking action aimed at the development and refinement of this branch of industry. Numerous studies are conducted, aimed at introducing new tech- 
nologies and solutions, both in terms of railway infrastructure and logistics management systems, as well as in traction vehicles themselves (Grebowski and Zielinska, 2015; Oleszak et al., 2013; Stypula; Stypula, 2009; Bruni et al., 2011; Connolly et al., 2014; Hecht 2009; Herwig and Bruhwiler, 2011; Jönsson et al., 2008, Kovalev et al., 2009; Mehrpouya and Ahmadian, 2009). Such actions directly translate into an increase in its effectiveness, safety, reduction of burden on the environment and society. This paper contains a report on the part of works conducted in the research and development project entitled "Analytical and experimental studies and determination of the structural features of components and assemblies in innovative structure of repaired wagons". This project is realized within the Program of Applied Research by Institute of Engineering Processes Automation and Integrated Manufacturing Systems of Silesian University of Technology together with consortium partners: company DB Schenker Rail Poland SA and Germaz. The main objective of the project is to develop a technology of modernization of freight wagons for the transport of coal and aggregates, through the use of innovative materials and technologies to repair this type of wagons during periodic repairs. Actions which have been undertaken within the project are to improve the operating conditions considered types of wagons by increasing their resistance to corrosion and freezes transported cargo to the shell of the body in the winter conditions, and thus an easier unloading (Wrobel et al., 2015; Baier and Zolkiewski, 2013; Placzek et al., 2015 a,b). An additional objective is also verification of strength of modernized carriages and an estimation of the possibility of reducing their weight, while maintaining or increasing the permissible load. One of elements of the project is also to develop a system for diagnosing the technical condition of the modernized shell of wagon body during operation. For this purpose the use of non-destructive testing methods of technical state of constructions will be used, including methods that use the analysis of dynamic response of the object. Therefore research is conducted which examines the possibility of use of the foils with piezoelectric properties as sensors used in the system of vibration measurement of tested items (Placzek et al., 2015a). These research efforts are a continuation of previous work related to the analysis of possibilities to use of composite materials as a part of the wagons boxes shell. Application of the composite panels to the freight wagon's body shell was proposed as the solution that can solve mentioned problems during exploitation of freight wagons (Wrobel et al., 2015; Baier and Zolkiewski, 2013; Placzek et al., 2015 a,b). The composite panels composed of fiberglass and epoxy resin were proposed. They will be mounted on the body shell using rivet nuts. What is more the body shell of the modernized freight wagon will be painted using an anticorrosion agent.

\section{A PROCESS OF QUALIFICATION TO REPAIR}

The main aim of the system designed for of inferred from the dynamic response of the wagon about the changes in its technical condition is to support a process of freight wagons qualification to repair. At this moment all freight wagons should be verified about their technical condition at a certain time interval. During the qualification process a qualifying protocol is used. In the DB Schenker Rail Poland SA Company in order to decide which elements of the freight wagon should be repaired or replaced by the new ones the protocol includes such data as:

- the type of the analysed freight wagon;
- the drawings of the analysed type of wagons including views of all side walls and the floor;

- the identification number of the wagon;

- the date and type of the previous repair and the date and type of the actual renovation;

- the table with listed elements of the wagon with the possibility to mark whether the item is suitable for repair, replacement, or whether it is in good technical condition;

- the signatures of the responsible people and the place for other notes.

During mentioned research work also a statistic analysis of data from qualifying protocols were analysed in order to verify if there are elements the most exposed to damage during standard exploitation of the freight wagons. Qualifying protocols from years 2012 up to 2014 were analysed. The number of analysed protocols was 298 for different types of freight wagons. Protocols were scanned and sorted taking into account types of freight wagons. Data from protocols were analysed in order to specify the percentage of repairs of individual wagons elements. Obtained results were presented on charts. In order to analyse date from protocols elements of the freight car's box were named and marked on the scheme of the freight wagon. In Fig. 1 names of the EAOS $1415 \mathrm{~A} 2$ and $\mathrm{A} 3$ wagon's elements designations are presented. The front of the wagon is indicated by the position of the brake cylinder.

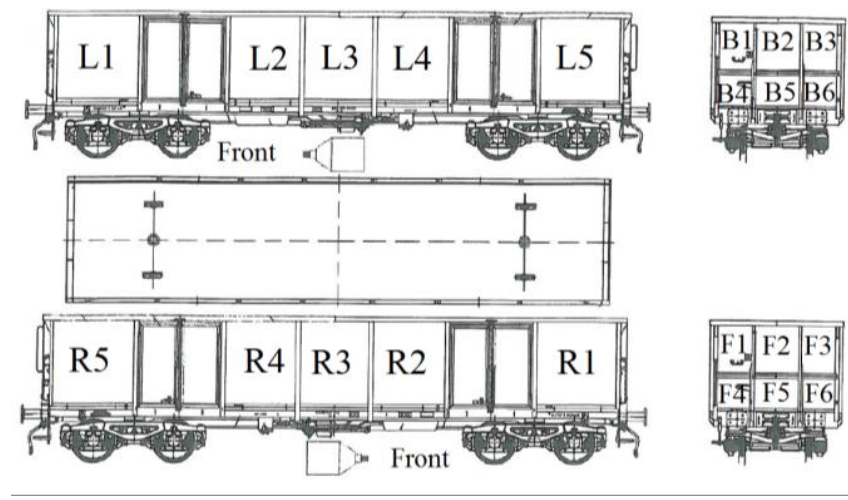

Fig. 1. Designation of EAOS $1415 A 2$ and $A 3$ wagon's elements, where symbols denotes: $L$ - the left side, $R$ - the right side, $\mathrm{F}-$ the front, $\mathrm{B}$ - the back of the wagon

The most important, taking into account assumption of modernization of the freight wagons using composite panels mounted to the wagon's shell of the body, is the information about the damage of its elements. Percentage of repairs of individual elements of the shell of the body is presented in Fig. 2 .

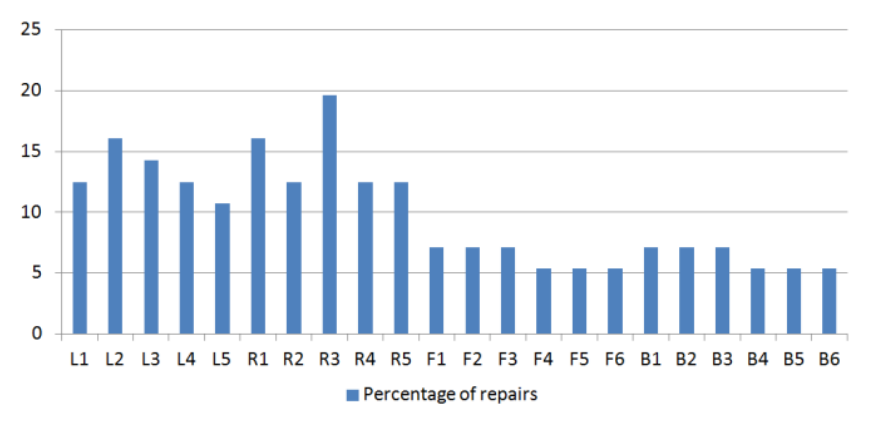

Fig. 2. Percentage of individual elements repairs of the body shell of freight wagon EAOS 1415 
Presented results of qualifying protocols analysis shows that there are some different between percentage of repairs of different elements of the freight wagons body shell. In case of type EAOS 1415 freight wagons it can be noticed that elements of the side walls were repaired more often than elements of the front wall and the back wall. What is more it can be noticed that even simetrical elements of the freight wagon (ex. L3 and R3) have different values of percentage of repairs. It can be explain by the random characteristic of the corrosion process of freight cars. It should be mentioned that in order to obtain more precise information about the process of damage of individual elements of the freight wagons body shell during their exploitation it is necessary to carry out a more accurate operational documentation starting from the production of wagons, which will take into account operating conditions of carriage, such as the type of transported cargo, as well as all carried out repairs.

The system that is to be developed should inform about destruction of the wagon's body shell during standard operation of the wagon. This should help to detect of major defects of wagon's body shell without transporting it to the service station. In order to develop such system it is necessary to verify the possibility of inference from the dynamic response of the wagon about the changes in its technical condition during its standard operation.

\section{Channel 1}
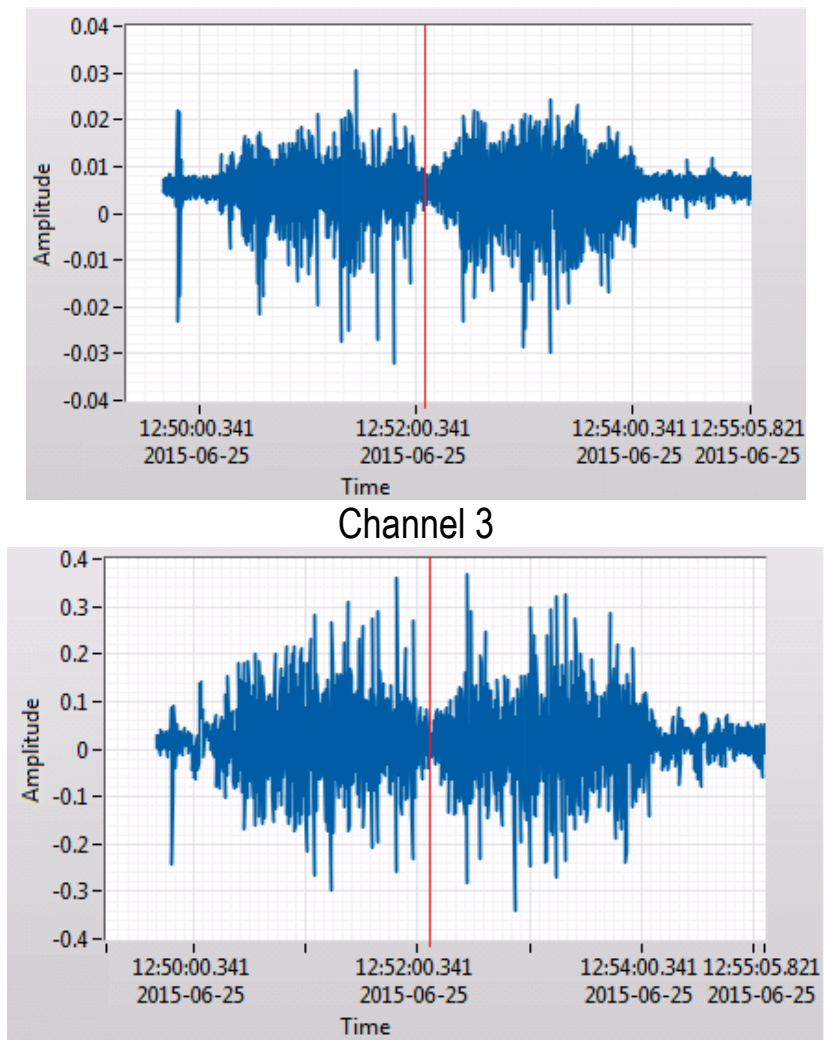

\section{MEASUREMENTS OF WAGON'S VIBRATION DURING OPERATION}

At the first step of the carried out tests measurements of the wagon's elements vibration during operation were carried out.

The object of research is the four axial freight wagon of ordinary type Eaos 1415-A3 production BREC Belgium. It is presented in Fig. 3. It was being taken into consideration because it is one of the most popular types of wagons designed to unload with the use of tipplers.

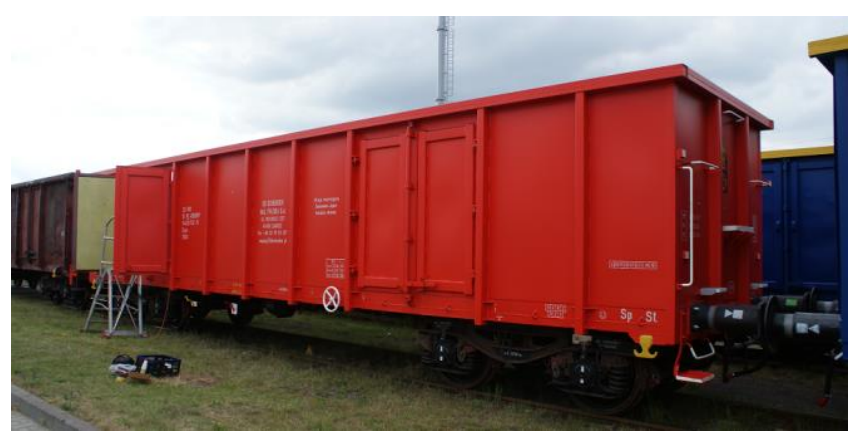

Fig. 3. The considered type of freight wagon

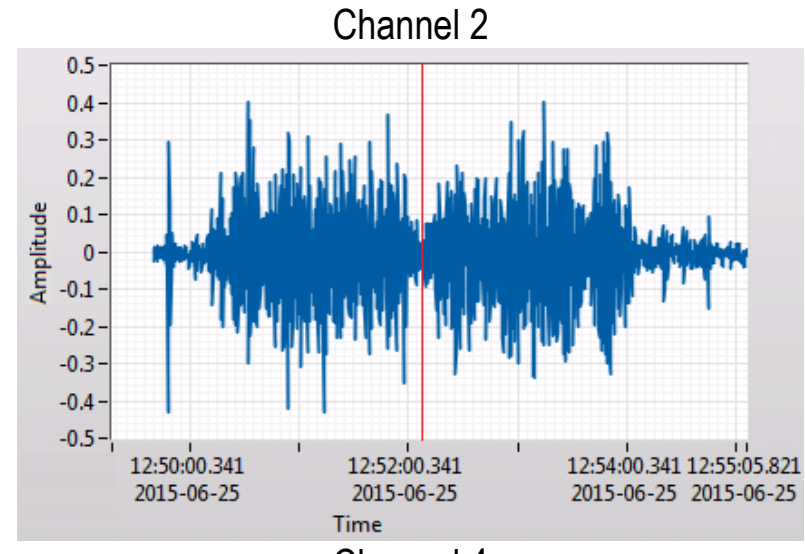

Channel 4

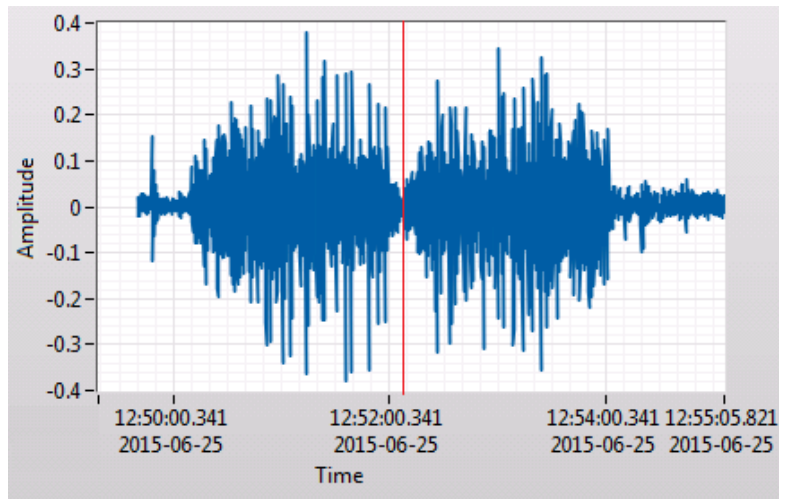

Fig. 4. The measured profile of excitations (empty wagon and max. speed $10 \mathrm{~km} / \mathrm{h}$ )

Measurements were carried out using analogue input card type NI-9215 (http://www.ni.com/pdf/manuals/373779a_02.pdf) and portable device NI cDAQ-9191 (http://www.ni.com/datasheet/ pdf/en/ds-371) - the compact 1-Slot chassis which allows Send data to a host PC over Ethernet or IEEE 802.11 Wi-Fi. As sensor M-8514-P1 model of Macro Fiber Composite transducer was used with active area $85 \times 14 \mathrm{~mm}$ (http://www.smart-material.com/MFCproduct-main.html). Four sensors were glued on the surface of the frame and body shell of the tested wagon and connected to the portable measurement system for data acquisition. Measurements were carried out while the observed train was driving (locomotive and two wagons) with the maximum travelling speed about 
$10 \mathrm{~km} / \mathrm{h}$. The speed of the train was limited as a result of the applicable regulations because measurements were carried out in the area of the railway repair company. There was no agreement to conduct research during normal exploitation. The train was driving a section of about 400 meters, stopped and then it was returning. The test was repeated five times to verify the repeatability of the results. Measurements were made in repair facility of DB Schenker Rail Poland in Rybnik. Measurement points were as:

Channel 1 - the centre of the top band of the box, interior of the freight wagon;

Channel 2 - the support frame - the bottom surface of the main, outer beam of the frame;

Channel 3 - the support frame - the inner surface of the main, outer beam of the frame; beam.

Channel 4 - the support frame - side surface of the cross-

Measuring points were selected on centre points of main beams of the wagon's frame and on the centre of the top band of the box because it was predicted that deformation of surfaces of these elements of the wagon construction will be the biggest. This was important for the measurements using MFC piezoelectric
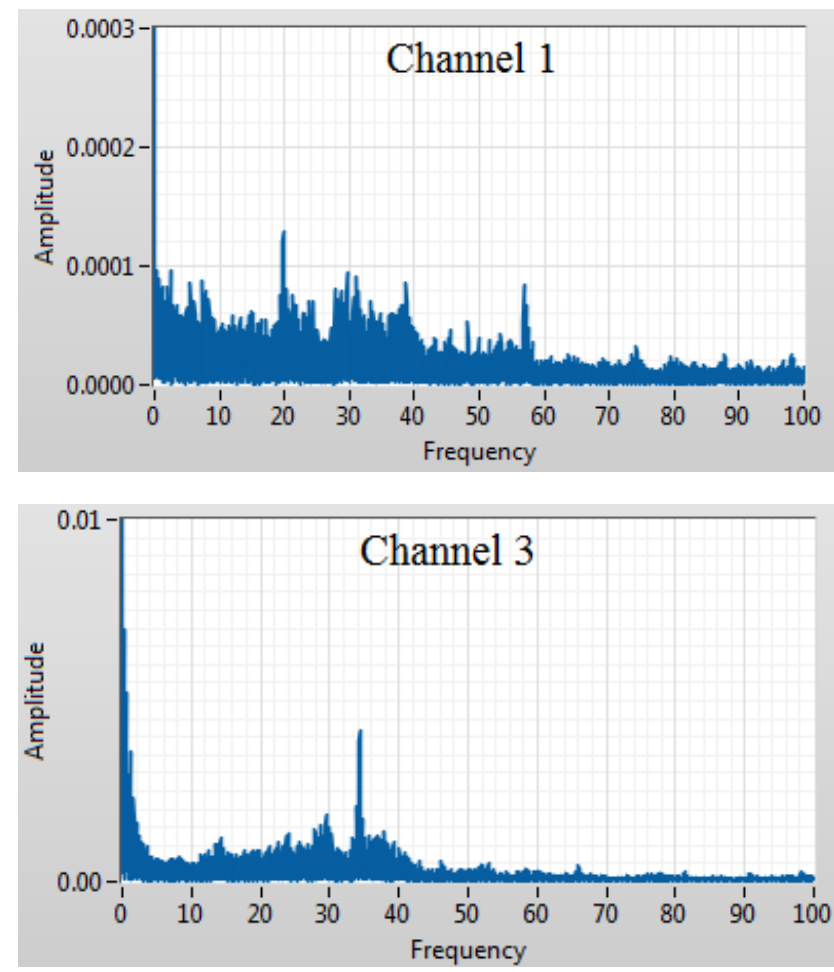

Fig. 5. An example of results of measured signal FFT analysis

The carried out measurements proved that results are repetitive and can be used in laboratory tests. During all measurements it could be observed that obtained results are repeatable (taking into account range of possible measurement errors) for all tested measuring points without influence of the weather conditions and temperature. It can be concluded that Macro Fiber Composite piezoelectric transducers proposed as sensors in developed system could be successfully used. They have a lot of advantages which causes that they can be proposed as a very good solution, for example they can be easily applied to the structure by gluing it to its surface. They are produced in the form of thin foils, so they also can be easily laminated into the structure of the composite panels that will be mounted in the modernized freight wagons. foils glued on surfaces of these elements.

The main aim of the measurements was to acquire a profile of excitations that are elements of the freight wagon exposed to during exploitation of the wagon. This profile was in the next step used in tests using laboratory stands in order to verify their dynamical response onto the excitation occurring during operation. In Fig. 4 examples of results of measurements obtained for the described channels are presented. The measured profile of excitations occurring during operation for empty wagon and maximal speed $10 \mathrm{~km} / \mathrm{h}$ for all measuring points is presented. The red vertical line separates two operating ranges: in the first range the wagon was pulled by the locomotive, while in the second one the wagon was pushed by the locomotive.

It can be observed that the electric voltage generated by the piezoelectric MFC sensors has not a high value but measured signal can be used in order to reproduce the extortion profile course during laboratory tests.

The measured signal was then analysed used Fast Fourier Transform to verify the resonance zones of the vibrating elements. An example of results of measured signal FFT analysis obtained using Hanning window is presented in Fig. 5.
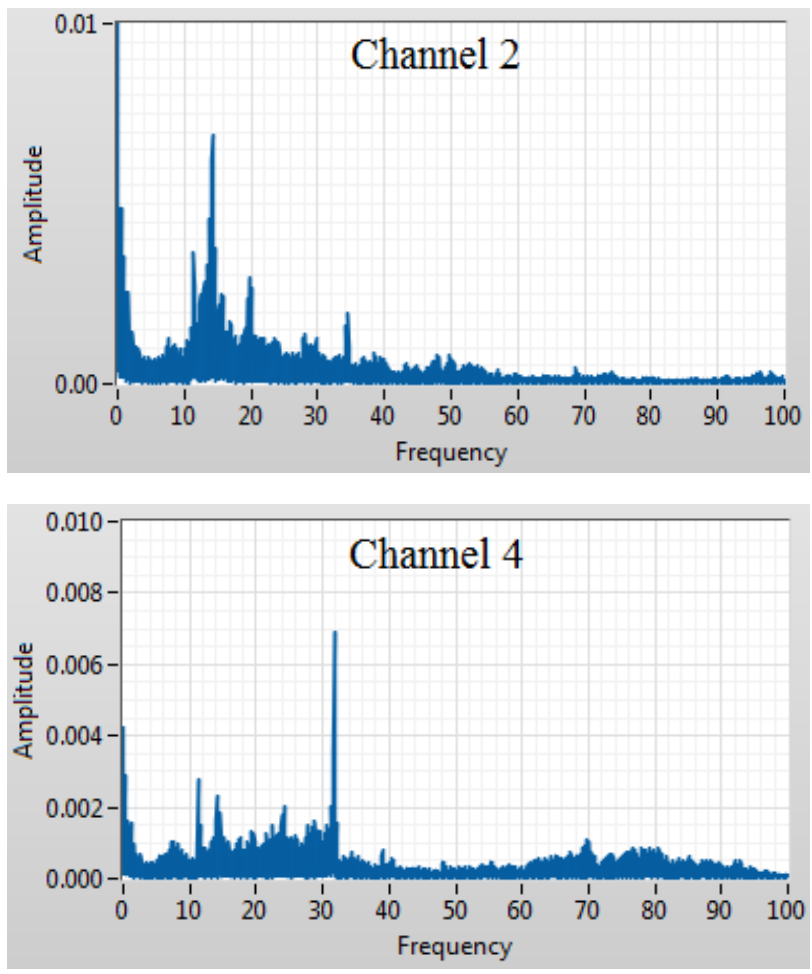

What is more they are resistant to weather conditions and do not require additional power source. What is more, for a low frequencies measurments the store \& hold amplifier can be used with the MFC transducers what can improve precision of measurements (http://www.smart-material.com/MFC-product-main.html).

\section{MEASUREMENTS USING LABORATORY STAND}

The composite panels composed of fiberglass and epoxy resin were proposed. They will be mounted on the body shell using rivet nuts. What is more the body shell of the modernized freight 
wagon will be painted using an anti-corrosion agent. Application of the new materials in the considered freight wagons is connected with the need to conduct a series of studies of the proposed materials properties, taking into account their strength analysis, abrasion resistance, fatigue resistance, and other properties important because of the working conditions in the modernized freight wagons. Very important property that has strong influence onto the safety of the modernized freight wagons exploitation is also the verification of the new elements flammability. Results of those studies were presented in other papers (Wrobel et al., 2015; Baier and Zolkiewski, 2013; Placzek et al., 2015 a,b).

a)

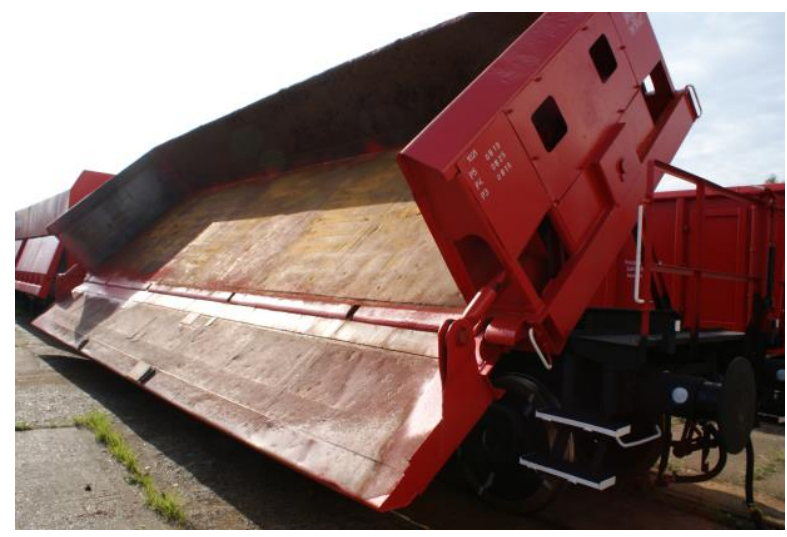

b)

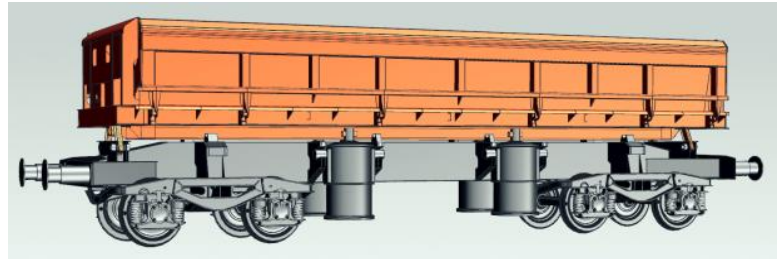

Fig. 6. The type of type $418 \mathrm{~V}$ freight wagon (a) and its CAD model (b)

a)

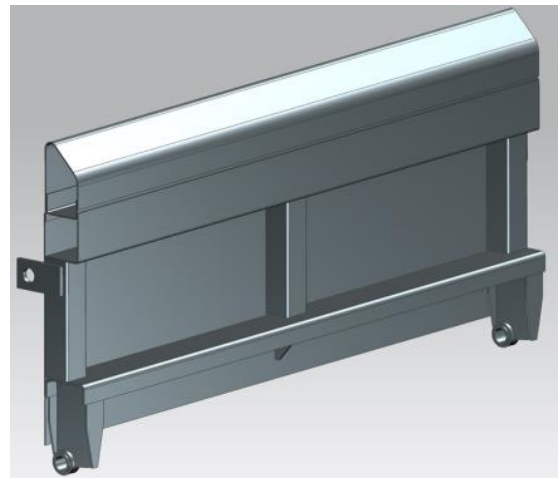

b)

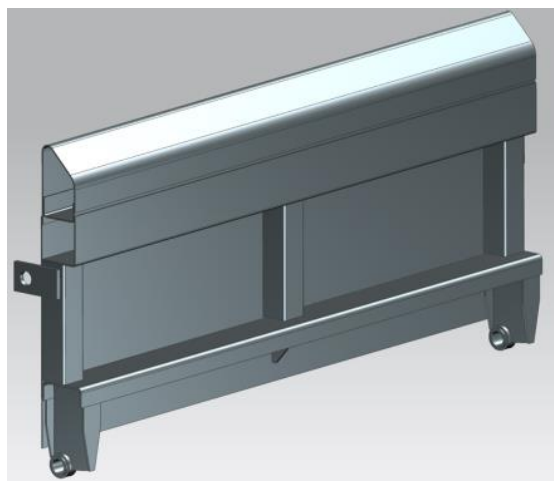

Fig. 7. The $C A D$ model of the tested piece of $418 \mathrm{~V}$ freight wagon's side (a) and The CAD model of the laboratory stand (b)
At the next step the measured profile of vibration excitation that is a result of the operation of the freight wagon was used for tests on a created laboratory stand. The laboratory stand was prepared for strength analysis of a side of a hopper freight wagon type 418V. This type of wagon is presented in Fig. 6.

In Fig. 7a) a CAD model of the tested piece of the freight wagon's side is presented. In carried out measurements the laboratory stand for strength analysis of the $418 \mathrm{~V}$ freight wagon side element was used. A CAD model of the stand with the analysed wagon's side is presented in Fig. $7 \mathrm{~b}$ ). The side's element was prepared in $1: 2$ scale.

During measurements the freight wagon's side was excited using vibration test system produced by TIRA GmbH. As sensors also Macro Fiber Composite M-8514-P1 piezoelectric transducers were used and connected with analogue input cards and portable device NI CDAQ-9191. Measurements were carried out with and without composite panels mounted to the wagon's side using rivet nuts. The aim of this work was to verify if it is possible to detect the presence of composite panels mounted to the tested wagon's side element. The tested element was excited using electrodynamic shaker and vibration profile obtained during the measurements carried out on the real freight wagons. Vibrations of the analysed side were measured in four measuring points using MFC piezoelectric transducers. The MFC sensors were glued to the surfaces of the side's frame as well as to the sheet metal fill. Measuring points were selected on centre points of main beams of the wagon's side model and on the centre point of the composite panel. It was predicted that deformation of surfaces of these elements of the wagon's side model will be the biggest.

\section{RESULTS}

Results obtained for measuring points on the side's frame and on the sheet metal fill for the wagon's side model with and without composite panels are presented in this work. As the next step of the carried out analysis the Fast Fourier Transform of measured signals was carried out in order to verify if any change in the obtained results can be observed. Results of the FFT analysis of measured signals obtained for the system with and without composite panels are juxtaposed in Fig. 9 up to frequency $200 \mathrm{~Hz}$. The red line denotes results obtained for the model of the freight wagon's side without any additional element while the blue line denotes results for the model with composite panels. The panels were made of fiberglass and epoxy resin with thickness $6 \mathrm{~mm}$. They were mounted using rivet nuts.

It can be noted that for both measuring channels the analysed system has higher values of the amplitude for all frequencies of vibrations in case when there is no composite panels mounted. Such results can be very useful for the algorithm that will be use in the system designed for modernized freight wagons technical condition monitoring during their exploitation. There may be a possibility to detect destruction of the mounted composite panels by the system on the basis of measured signals from applied piezoelectric foils. The system should also detect if the freight wagon is loaded or it is used empty and inform about its status using GPS system. Such information can be very useful for better managing of freight wagons and for data collecting in the system that should record the history of the wagons exploitation. 
a)

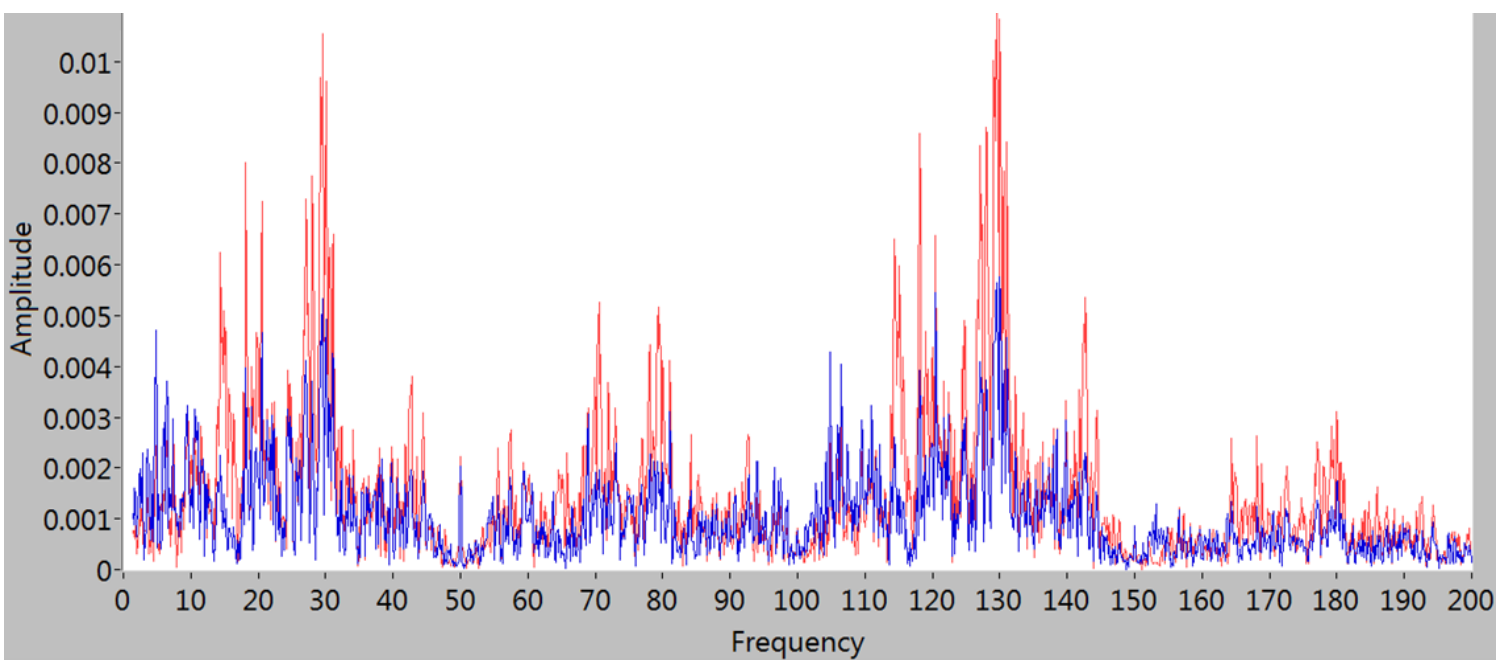

b)

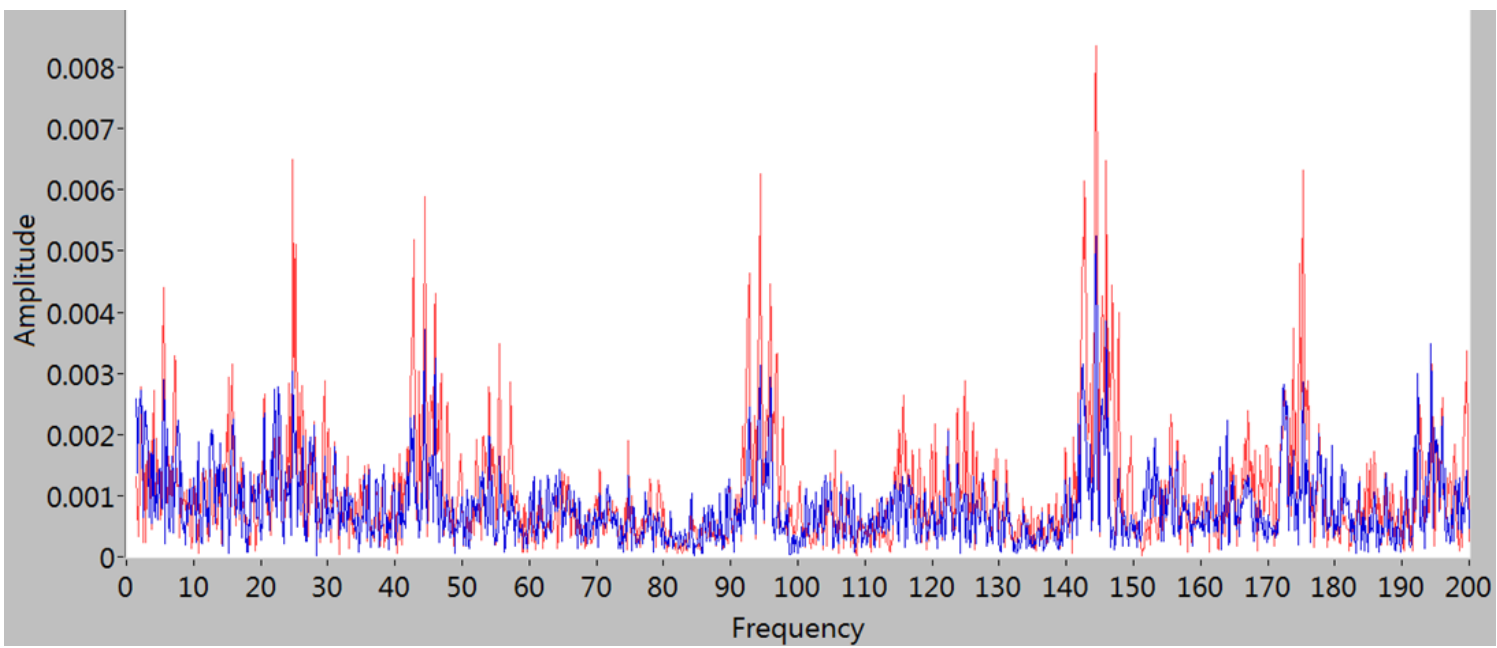

Fig. 9. Results of the FFT analysis of measured signals juxtaposed for the system witch (the blue line) and without (the red line) composite panels for channel 1 (a) and channel 2 (b)

\section{CONCLUSIONS}

Carried out tests and measurements on freight wagons as well as using laboratory stands proved that Mecro Fiber Composite piezoelectric transducers can be successfully used as sensors for measurements of freight wagon's elements vibrations. They can be easily applied to the surface of monitoring structure as well as laminated in composite panels that will be used during proposed freight wagons modernization. What is more they can be easily protected to weather conditions.

During carried out tests on the real freight wagon the speed was limited and it was lower than speed of a train during its standard exploitation. It caused that values of measured signals were lower than they can be predicted in application on freight wagons for standard operation. However, using measured profile of excitation it was possible to infer about the status of the laboratory stand. Taking into account obtained results of laboratory tests it was possible to conclude whether composite panels are connected to the tested model of the freight wagon's side or they are lacked. Obtained results allows to conclude that proposed method of control of technical condition of modernized freight wagons may be a good solution.

In further research more measurements on laboratory stands and especially on real objects during their standard exploitation will be carried out in order to verify repeatability of the obtained results and to take into account influence of various conditions such as weather conditions, deformations of the wagon's body shell that are results of its exploitation, mainly unloading using excavators etc. The influence of changes in monitored system created during its exploitation appears as a main problem which may result in system malfunction.

\section{REFERENCES}

1. Baier A., Zolkiewski S. (2013), Initial research of epoxy and polyester warp laminates testing on abrasive wear used in car sheathing, Eksploatacja i Niezawodnosc - Maintenance and reliability, 15(1), 37-43.

2. Banas W., Sekala A., Gwiazda A., Foit K., Hryniewicz P., Kost G. (2011), The modular design of robotic workcells in a flexible production line, IOP Conf. Series: Materials Science and Engineering, 95, 012099.

3. Bruni S., Vinolas J., Berg M., Polach O., Stichel S. (2011), Modelling of suspension components in a rail vehicle dynamics context, Vehicle System Dynamics, 49(7), 1021-1072.

4. Buchacz A., Banas W., Placzek M. (2015), Influence of the excitation parameters of the mechanical subsystem on effectiveness of energy harvesting system, IOP Conference Series-Materials Science and Engineering, 95, 012052.

5. Buchacz A., Galeziowski D. (2012), Synthesis as a designing of mechatronic vibrating mixed systems, Journal of Vibroengineering, 14(2), $553-559$. 
6. Buchacz A., Placzek M., Wrobel A. (2014), Modelling of passive vibration damping using piezoelectric transducers - the mathematical model, Eksploatacja i Niezawodnosc - Maintenance and Reliability, 16(2), 301-306.

7. Connolly D.P., Kouroussis G., Giannopoulos A., Verlinden O., Woodward P.K., Forde M.C. (2014), Assessment of railway vibrations using an efficient scoping model, Soil Dynamics and Earthquake Engineering, 58, 37-47.

8. Grebowski K., Zielinska M. (2015), Modelling of dynamic interactions of Pendolino train type on structures of historic railway bridges in Poland, Przeglad Budowlany, 1, 27-32.

9. Gwiazda A., Herbuś K., Kost G., Ociepka P. (2015), Motion analysis of mechatronic equipment considering the example of the Stewart platform, Solid State Phenomena, 220/221, 479-484.

10. Hecht M. (2009), Wear and energy-saving freight bogie designs with rubber primary springs: principles and experiences, Proceedings of the Institution of Mechanical Engineers Part F: Journal of Rail and Rapid Transit, 223(2), 105-110.

11. Herwig A., Bruhwiler E. (2011), In-situ dynamic behaviour of a railway bridge girder under fatigue causing traffic loading, Proceedings of the 11th International Conference on Applications of Statistics and Probability in Civil Engineering, 389-395.

12. Jamroziak K., Bocian M., Kulisiewicz M. (2013), Effect of the attachment of the ballistic shields on modelling the piercing process, Mechanics, 19(5), 549-553.

13. Jamroziak K., Bocian M., Kulisiewicz M. (2013), Energy consumption in mechanical systems using a certain nonlinear degenerate mode, J. of Theor. and Appl. Mechanics, 51(4), 827-835.

14. Jönsson P.A., Stichel S., Persson I. (2008), New simulation model for freight wagons with UIC link suspension, Vehicle System Dynamics, 46, 695-704.

15. Klarecki K., Rabsztyn D., Hetmanczyk M. (2015), Analysis of pulsation of the sliding-vane pump for selected settings of hydrostatic system, Eksploatacja i Niezawodnosc - Maintenance and Reliability, 17(3), 338-344.

16. Kovalev R., Lysikov N., Mikheev G., Pogorelov D., Simonov V., Yazykov V., Zakharov S., Zharov I., Goryacheva I., Soshenkov S., Torskaya E. (2009), Freight car models and their computer-aided dynamic analysis, Multibody System Dynamics, 22(4), 399-423.

17. Mehrpouya M., Ahmadian H. (2009), Estimation of applied forces on railway vehicle wheelsets from measured vehicle responses, International Journal of Vehicle Structures and Systems, 1(4), 104-110.

18. Monica Z. (2015), Optimization of the production process using virtual model of a workspace, IOP Conf. Series: Materials Science and Engineering, 95, pages.
19. Oleszak P., Ciesla J., Szaniec W. (2013), Study of the effects of side impacts on railway viaduct lying on the arc, Budownictwo i Architektura, 12(2), 47-54 (in Polish).

20. Paprocka I., Kempa W., Grabowik C., Kalinowski K. (2014), Predictive and reactive scheduling for a critical machine of a production system, Advanced Materials Research, 1036, 909-914.

21. Placzek M. (2015), Modelling and investigation of a piezo composite actuator application, International Journal of Materials \& Product Technology, 50(3-4), 244-258.

22. Placzek M., Buchacz A., Wrobel A. (2015a), Use of piezoelectric foils as tools for structural health monitoring of freight cars during exploitation, Eksploatacja i Niezawodnosc - Maintenance and Reliability, 17(3), 443-449.

23. Placzek M., Wrobel A., Baier A. (2015b), Computer-aided strength analysis of the modernized freight wagon, IOP Conference SeriesMaterials Science and Engineering, 95, 012042.

24. Rusinski E., Dragan S., Moczko P., Pietrusiak D. (2012), Implementation of experimental method of determining modal characteristics of surface mining machinery in the modernization of the excavating unit, Archives of Civil and Mechanical Engineering, $12,471-476$.

25. Stypula K. (2009), Selected problems of surface building protection against vibrations generated by underground communication, Gornictwo i Geoinzynieria, 3(1), 351- 362 (in Polish).

26. Tuma J. (2009), Gearbox Noise and Vibration Prediction and Control, Int. J. of Acoustic and Vibration, 14(2), 99-108.

27. Tuma J., Simek J., Skuta J., Los J., Zavadil J. (2011), Active Vibration Control of Hydrodynamic Journal Bearings, Springer Proceedings in Physics, 139, 619-624.

28. Wrobel A., Placzek M., Buchacz A., Majzner M. (2015), Study of mechanical properties and computer simulation of composite materials reinforced by metal, International Journal of Materials \& Product Technology, 50(3-4), 259-275.

29. http://www.ni.com/pdf/manuals/373779a_02.pdf (Access 22.03.2017)

30. http://www.ni.com/datasheet/pdf/en/ds-371 (Access 22.03.2017)

31. http://www.smart-material.com/MFC-product-main.html (Access 22.03.2017)

The work was carried out under the project number PBS2/A6/17/2013 agreement implemented under the Applied Research Program, funded by the National Centre for Research and Development. 